\title{
Acciones de difusión de las Ciencias de la Tierra en Argentina hoy
}

\author{
Leonor Bonan \\ Instituto de Investigación en Enseñanza de \\ las Ciencias CEFIEC, Facultad de Ciencias \\ Exactas y Naturales, Universidad de \\ Buenos Aires. \\ Ibonan@de.fcen.uba.ar.
}

\begin{abstract}
Actions for Diffusion of EARth Sciences KnOWledge in Argentina Nowadays. This paper describes some resources for diffusion of scientific knowledge of the Earth Sciences, in the current Argentina society. They are contextualized in a data selection from the history of the Earth Sciences in the local context and in the current science policy. Finally, we examine its scopes and limitations, specially from the perspective of science education and popularization of scientific knowledge. Citation: Bonan L. 2014. Acciones de difusión de las ciencias de la tierra en argentina hoy. Terræ Didatica, 10(3):250-259. http://www.ige.unicamp.br/terraedidatica/.
\end{abstract}

KEYWORDS: Science Policy, popularization of scientific knowledge, Earth Science.

RESUMEN Este trabajo describe algunos recursos para la difusión del conocimiento científico asociado con las Ciencias de la Tierra, destinados a la sociedad argentina actual. Se los contextualiza a la luz de una selección de datos de la historia de las Ciencias de la Tierra en el contexto local $\gamma$ de la política científica actual. Por último, se reflexiona sobre sus alcances y limitaciones, en especial, desde la perspectiva de la popularización del conocimiento científico y de la educación en ciencias.

PALABRAS CLAVE: Política Científica, popularización del conocimiento científico, Ciencias de la Tierra. 


\section{1.- Introducción}

Actualmente en la República Argentina se realizan diferentes emprendimientos para difundir las Ciencias de la Tierra en la sociedad, algunos de ellos en la educación formal y otros en la educación no formal. Sin embargo, estas acciones ocurren en un momento particular que depende de factores políticos, sociales y económicos, y estos de su historia. Es importante visibilizar que el contexto de producción de la ciencia se rige por políticas científicas y estas de la política que aplica un gobierno. También es importante destacar que la ciencia es un producto histórico y social.

El conocimiento asociado con las Ciencias de la Tierra ha adquirido trascendencia especial en los últimos años en Argentina, a partir del crecimiento de la explotación minera y de la recuperación de la empresa petrolera de bandera YPF. Estas actividades se vinculan con la producción de conocimiento y con su enseñanza pues exigen formar técnicos, profesionales y científicos en disciplinas afines a las Ciencias de la Tierra. Esta área es muy poco conocida por el público, en general, y tiene una presencia muy marginal en todos los niveles del sistema educativo. La conjunción de estas circunstancias desataron un interés especial en dar a conocer diferentes aspectos de este campo de conocimiento y esto, a su vez, condujo a desarrollar acciones de popularización más allá de otras acciones iniciadas en el sistema educativo.

El sistema científico argentino fue construido a la luz de su historia, atravesando períodos de crecimiento sostenido y otros de vaciamiento generalizado. La política implementada por los gobiernos de los últimos 10 años considera que el sistema científico es estratégico y promueve con énfasis sus vínculos con el desarrollo tecnológico y la innovación productiva. En este contexto, la educación y la popularización del conocimiento científico son consideradas de suma relevancia para captar gentte que materialice estas políticas.

En este contexto, este trabajo presenta una serie de acciones de popularización de la ciencia y de la educación científica asociadas con las Ciencias de la Tierra. Para ello, se describen brevemente los hitos de la historia de la ciencia argentina que permitieron generar la comunidad científica asociada a esta área y el contexto político en relación con la difusión de las Ciencias de la Tierra en la sociedad argentina actual. Se describe brevemente el soporte teórico que vincula las acciones de popularización y educación científicas con sus finalidades y medios de materialización. Por último, se enuncian algunas reflexiones.

\section{2.- Algunos datos de la historia de las Ciencias de la Tierra en Argentina}

Las Ciencias de la Tierra, a los fines de este escrito, evocan aquellas disciplinas que se han desarrollado en el país: geología, meteorología y oceanografía, tanto desde el punto de vista científico como tecnológico, a través de su anclaje institucional. El desarrollo de esta área se remonta al inicio de la historia de la patria. Algunos de sus antecedentes no son exclusivos de las Ciencias de la Tierra sino que refieren al desarrollo del sistema científico argentino aunque otros que aquí expondremos le son propios.

Según Ramos (Ramos 2011) desde la primera revolución patria pueden identificarse antecedentes tendientes al desarrollo de las Ciencias de la Tierra en Argentina. Inicialmente, la ruptura colonial marcó la necesidad imperiosa de fundar nuevas instituciones en las que se desarrollaran las ciencias. Posteriormente, hubo gobiernos que realizaron grandes esfuerzos en este sentido, creando algunas instituciones que permanecen hasta el presente y otros (especialmente las dictaduras militares y los gobiernos liberales y neoliberales) han materializado políticas tendientes a su vaciamiento.

Otra contribución temprana importante al desarrollo de esta área que no se puede omitir, se relaciona con la exploración realizada por viajeros extranjeros, como Alcide d'orbigny y Charles Darwin. Alcide d'orbigny estuvo en Sudamérica entre 1826 y 1833 y Darwin en 1831. Estos naturalistas relevaron y sistematizaron información científica local de importancia, lo que constituyó un gran aporte a diferentes áreas de las ciencias naturales en Argentina.

Desde el punto de vista institucional, en 1824 se creó el Museo Nacional de Ciencias Naturales y, a partir de la sanción de la Constitución Nacional en 1853, se crearon nuevas instituciones de carácter científico. En 1865, llegaron al país los primeros especialistas que enseñaron Ciencias de la Tierra en Argentina. Vinieron de Italia y Alemania, dando lugar a sistematizar su enseñanza en la universidad. Primero se enseñó en la Universidad Nacional de Buenos Aires, y unos años más tarde en la Universidad Nacional de Córdoba. Esta acción educativa fue cimiento para la creación de una serie de instituciones durante el siglo $\mathrm{XX}$, que expandieron el desarrollo de las Ciencias de la Tierra a todo el país (Ramos 2011). Nombraremos algunas de ellas: en 1871 se inauguró el Observatorio Astronómico de 
Córdoba, en 1872 la Sociedad Científica Argentina y la Oficina Meteorológica Argentina, en 1873 se estableció la primera Red de Estaciones Meteorológicas, en 1904 la Dirección Nacional de Minería y Geología (que tuvo diferentes denominaciones: Dirección de Minas y Geología, Instituto Nacional de Geología y Minería y actualmente Servicio Geológico Minero Argentino -SEGEMAR- ); en 1907 se encontró petróleo en suelo argentino y en 1922 se fundó Yacimientos Petrolíferos Fiscales (YPF). En 1942 se fundó Yacimientos Carboníferos Fiscales (YCF), en 1950 la Comisión Nacional de Energía Atómica (CNEA), en 1945 la Asociación Geológica Argentina (denominada inicialmente Sociedad Geológica Argentina), en 1951 el Instituto Antártico Argentino, en 1958 el Consejo Nacional de Investigaciones Científicas y Técnicas (CONICET), entre las instituciones más importantes. La creación de estas instituciones es un buen indicador del desarrollo histórico de esta área científico-tecnológica en Argentina.

Además, desde la llegada de los primeros académicos del área, de manera ininterrumpida y en forma creciente se desarrolló la enseñanza de las Ciencias de la Tierra en la universidad, expandiéndose a diferentes lugares del país. Sin embargo, es necesario señalar que la expansión refiere a la geología y a algunas ingenierías asociadas con la actividad petrolera y minera. Tanto la meteorología como la oceanografía han tenido desarrollos más acotados siendo que actualmente se consideran áreas de vacancia.

La década de 1990 se caracterizó por reducir a su mínima expresión los aportes destinados al desarrollo de la actividad científica. En estas circunstancias, el sistema científico tocó el umbral de la subsistencia y obligó a parte de la comunidad científica a emigrar-hecho conocido popularmente como la fuga de cerebros--. Todos estos antecedentes dan una idea de cómo se constituyeron las Ciencias de la Tierra en Argentina, punto de partida para su situación actual.

\section{3.- Contexto político actual de difusión de las Ciencias de la Tierra}

El gobierno que asumió en 2003 apreció la base científica argentina construida a lo largo de casi 200 años de historia, supo de su reconocimiento internacional y consideró beneficioso para el país vincularla con su matriz productiva y su desarrollo tecnológico. La acción más importante en este sentido fue la creación, en 2007, del Ministerio de Ciencia, Tecnología e Innovación Productiva, dándole categoría ministerial a lo que antes era una secretaría, proponiendo "orientar la ciencia, la tecnología y la innovación al fortalecimiento de un nuevo modelo productivo que genere mayor inclusión social y mejore la competitividad de la economía argentina, bajo el paradigma del conocimiento como eje del desarrollo" (Argentina 2007). Este gran objetivo integra diferentes componentes al desarrollo científico, otorgando un lugar de relevancia a su aplicación a la matriz productiva, generando la necesidad de formar científicos, ingenieros y técnicos afines a las actividades científicas y profesionales que el modelo político pretende desarrollar y aplicar. En especial, este nuevo ministerio se propuso generar e implementar políticas públicas que conllevaran la popularización del conocimiento científico, dando lugar al desarrollo de variadas propuestas de educación no formal, algunas de ellas vinculadas con las Ciencias de la Tierra (Megamuestra Tecnópolis, Tecnópolis TVy Semana de las Ciencias y la Tecnología).

Por otra parte, el Ministerio de Educación de la Nación, en coherencia con la política desarrollada por el Ministerio de Ciencia, Tecnología e Innovación Productiva, consideró que la enseñanza de las ciencias naturales era un área estratégica para el desarrollo del país, por lo que realizó un relevamiento de su estado del arte, con el fin de implementar políticas educativas específicas que mejoraran la enseñanza de las ciencias naturales, en general, en el país. Entre ellas, algunas tendientes a promover la enseñanza de las Ciencias de la Tierra en la educación formal. En especial, el Programa ConectarIgualdad, cuyo objetivo más destacado es la inclusión social a través de las nuevas tecnologías, considera la escuela como un medio privilegiado para democratizar el acceso al conocimiento. Este programa se propone preparar al sistema educativo para formar a los estudiantes en la utilización comprensiva y crítica de las nuevas tecnologías.

Un hecho que en apariencia no tiene nada que ver es la sanción de la Ley 26.522 de Servicios de Comunicación Audiovisual, que promueve la democratización y la universalización de las nuevas tecnologías de la información y la comunicación. Los alcances de esta ley condujeron a las autoridades a generar contenidos audiovisuales nacionales dado que la ley así lo exige. Entre ellos algunos relacionados con las Ciencias de la Tierra y, en muchos casos, en coordinación con los objetivos de ambos ministerios. 
La política científica conducida por los gobiernos argentinos desde el año 2003 resultó especialmente propicia para la popularización del conocimiento científico, en especial, el que refiere a las Ciencias de la Tierra.

\section{4.- Contexto teórico de la popularización de la ciencia y de la educación científica}

Varios pueden ser los motivos de un país para que decida implementar una política pública de popularización y educación del conocimiento científico, entre ellos: promover la prosperidad nacional y el ejercicio de la ciudadanía, contribuir al desempeño económico de las personas, fundamentar la toma de decisiones personales --en relación con la salud o la alimentación, por ejemplo-- y, también, divulgar la ciencia entre los propios técnicos y científicos (Castro Moreira 2006). Cuando la popularización de la ciencia y la tecnología se vuelve una política pública se constituye en un medio de inclusión social y, por tanto, de disminución de la desigualdad. Las sociedades más igualitarias evidencian un mejor rendimiento de los estudiantes en el aprendizaje de las ciencias (Sahlberg 2013). El proceso de popularización contribuye a que el conocimiento científico y tecnológico sea un componente central de la cultura, de la conciencia social y de la inteligencia colectiva, así como a la efectiva integración cultural, étnica, lingüística, social y económica. (Merino y Giamello 2000).

En los últimos años, existe una multiplicación de contextos de aprendizaje que amplían la mirada más allá del contexto escolar, entendiendo que la popularización del conocimiento científico es una forma de educación no formal que asume diferentes soportes para transmitir información de manera intencional: medios de comunicación, centros y museos de ciencia, programas de extensión universitaria, eventos de divulgación, entre otros. Pero para lograr una popularización científica eficaz es necesario expresar de manera explícita qué ciencia popularizar, con qué profundidad, qué aspectos, a quiénes estará dirigida, por cuánto tiempo se llevará a cabo la propuesta y, lo que es muy importante, con qué objetivos.

Los emprendimientos de popularización de la ciencia y la tecnología destinados a la sociedad en general, pueden potenciarse en interacción con las acciones que se dan al interior de la educación formal. Existe un gran número de instancias de promoción de la enseñanza de las ciencias, en particular de las Ciencias de la Tierra, en los diferentes niveles del sistema educativo, que exceden los alcances de este trabajo. Sin embargo, para que estas producciones se conviertan en aprendizajes es necesario reflexionar sobre los fines de la educación científica, con el interés de conectar la ciencia con sus aplicaciones tecnológicas y los fenómenos que permean la vida cotidiana, abordar el estudio de aquellos conocimientos que tengan relevancia social, profundizar las implicaciones sociales y éticas de la ciencia y fomentar una comprensión de la naturaleza de la ciencia y del trabajo científico (Sanmartí 2002). Este enfoque y otros compatibles (Gil Pérez y Vilches 2006; Massarini et al 2007) ampliaron los límites de la enseñanza de las ciencias naturales, visibilizando los aspectos relativos al ejercicio de la ciudadanía en una sociedad democrática, pensando en una educación científica para la acción social. Esta apertura de la educación científica conecta los aspectos conceptuales con las finalidades de su enseñanza, y a estas con la vida personal y social de los sujetos que aprenden, aspectos en común con la popularización de la ciencia. Las finalidades de las acciones de popularización se aprecian por lo que exhiben pero también por lo que ocultan.

\section{5.- Soportes para la difusión de las Ciencias de la Tierra}

La política científica implementada en los últimos años produjo diferentes acciones materiales o eventos de popularización científica, como así también materiales didácticos relacionados con las ciencias. En este apartado haremos un paneo de algunos de estos emprendimientos, haciendo énfasis en algún aspecto en relación con qué conocimientos de Ciencias de la Tierra se difunden y cómo se lo hace, es decir, con qué soporte se materializa la acción de difusión. Todas las acciones tienen páginas de internet asociadas a través de las que se puede ampliar la información aquí brindada que, desde ya, será muy escueta.

\section{1.- Megamuestra Tecnópolis}

Tecnópolis es una gran muestra de ciencia, tecnología, industria y arte que depende de manera directa de la Presidencia de la Nación. Se desarrolla en Villa Martelli, en las cercanías de la Ciudad de Buenos Aires, ocupando una superficie de 60 hectáreas. 


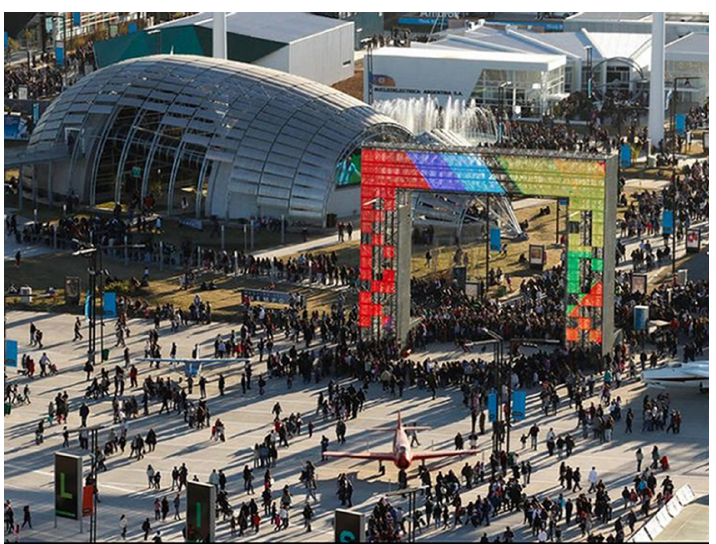

Figura 1. Tecnópolis: Megamuestra de ciencia, tecnología, industria y arte.

Se realizó por primera vez en 2011, llevando al momento 3 ediciones. Está abierta al público general, es de entrada libre y gratuita y promueve y apoya la visita de establecimientos escolares de todo el país. Cada edición se prepara en el primer semestre del año y abre sus puertas al público desde agosto hasta diciembre aproximadamente. Tiene un sitio de internet asociado que se actualiza permanentemente: http://tecnopolis.ar/2013/.

El diseño de la muestra contempla la información disponible en su página de internet. Se sugiere a los visitantes navegar la página antes de realizar la visita dado que allí hay información sobre lo que la muestra ofrece, dando la posibilidad de realizar recorridos temáticos o bien seleccionar qué stands visitar personalmente. Es tan grande la muestra que recorrerla en su totalidad requiere varias visitas.

Para tener una idea del tipo de propuestas que ofrece Tecnópolis expondremos algunos ejemplos de propuestas de popularización de las Ciencias de la Tierra.

\section{Paleontología}

Esta exposición se realiza al aire libre. Es un espacio ambientado en la era mesozoica en el que se encuentran 40 réplicas de dinosaurios robotizados a escala real.

http://tecnopolis.ar/noticiasdetecnopolis/ciencia/ un-parque-jurasico-en-villa-martelli/

Esta propuesta ofrece un recorrido por las especies que habitaron la geografía argentina hace millones de años. Se puede encontrar las réplicas de fósiles que científicos argentinos descubrieron e ingresar a un cine 3D en el que se exhibe un video de casi 5 minutos en el que se describen algunos aspectos relativos a la paleontología en Argentina y a los dinosaurios que la habitaron. También se puede acceder a un espacio recreativo de excavación, donde los niños más chicos encuentran réplicas de fósiles.

http://tecnopolis.ar/2013/atracciones/paleontologia

Esta exposición genera gran atractivo a los visitantes por la grandilocuencia de la puesta en escena.

\section{La experiencia de ser minero}

Este espacio invita a recorrer una réplica de las galerías subterráneas de la mina más grande de la Argentina, Yacimientos Carboníferos de Río Turbio. Allí se puede apreciar cómo es el proceso de extracción del carbón y la generación de energía termoeléctrica.

http://tecnopolis.ar/noticiasdetecnopolis/generales/ la-experiencia-de-ser-minero/

Se presenta la actividad de un minero de manera superficial, sin exponer las grandes desventajas a las que están sometidos los operarios que la realizan, los pasivos ambientales de la actividad y los aspectos críticos del intercambio económico a nivel global pues nuestra región exporta materias primas e importa bienes con alto valor agregado.

\section{YPF, energía para transformar}

Este stand invita a conocer la exploración y extracción de hidrocarburos a bordo de un simulador que conduce a casi 1500 metros de profundidad. El objetivo central de esta propuesta es resaltar la importancia del petróleo y el gas para el crecimiento del país.

http://tecnopolis.ar/noticiasdetecnopolis/ciencia/ la-aventura-de-la-energia/

La propuesta no incluye el desarrollo de los contenidos científicos asociados con la extracción de hidrocarburos ni los efectos asociados con el cambio climático.

\section{Geología}

En este stand se exhibe cómo es el trabajo que realizan los espeleólogos, a través de réplicas de cuevas y cavernas. También se puede apreciar cómo es un volcán por dentro y experimentar cómo se siente el comportamiento del suelo ante un evento sísmico a través de un simulador de terremotos. http://tecnopolis.ar/2013/atracciones/geologia

\section{La nueva minería}

Esta propuesta persigue el objetivo de mostrar cómo son las nuevas tecnologías mineras que 
se están desarrollando en la provincia de San Juan. El stand presenta maquetas de proyectos mineros, transportadores a minas subterráneas, mapas topográficos, proyecciones audiovisuales en pantallas $360^{\circ}$ y abundante información sobre la importancia de la actividad minera. No se hace referencia a los pasivos ambientales de la actividad.

http://tecnopolis.ar/2013/atracciones/san-juan-nueva-mineria

De manera general, la megamuestra Tecnópolis exhibe fenómenos científicos o tecnolológicos de manera espectacular pero también acrítica.

Otros sitios de interés que propone Tecnópolis relacionados con las Ciencias de la Tierra son:

http://tecnopolis.ar/noticiasdetecnopolis/ciencia/ los-secretos-del-clima/\#.UslVS9LuI7N

http://tecnopolis.ar/noticiasdetecnopolis/generales/un-minero-con-historia/\#.UslVt9LuI7M http://tecnopolis.ar/noticiasdetecnopolis/ciencia/ paleoartista-un-oficio-en-extincion/

\section{2.- Canal de Televisión Tecnópolis TV}

Tecnópolis TV (TECTV) es una señal nacional de Televisión Digital Abierta dedicada a la ciencia y la tecnología. Su programación se trasmite durante las 24 horas, con contenidos de origen nacional e internacional. TECTV es el canal de televisión del Ministerio de Ciencia, Tecnología e Innovación Productiva y fue creado en el marco del Programa Federal de Televisión Digital Terrestre, programa que está asociado con la sanción de la Ley de Servicios de Comunicación Audiovisual.

La finalidad de la señal es mostrar a la ciencia como una actividad generadora de crecimiento económico, capaz de mejorar la calidad de vida de todos los ciudadanos. El canal presenta en su programación series de ficción, documentales, entrevistas, biografías y microprogramas. Sus objetivos son: promover la ciencia, la tecnología, la innovación productiva, los emprendimientos y la actividad industrial; despertar vocaciones científicas y técnicas; e incentivar en los jóvenes el interés por resolver cuestiones de la vida diaria a través del conocimiento científico. Se puede acceder a su programación on line a través de su página de internet: http://www.tectv.gob.ar/ A modo de ejemplo, seleccionamos algunos

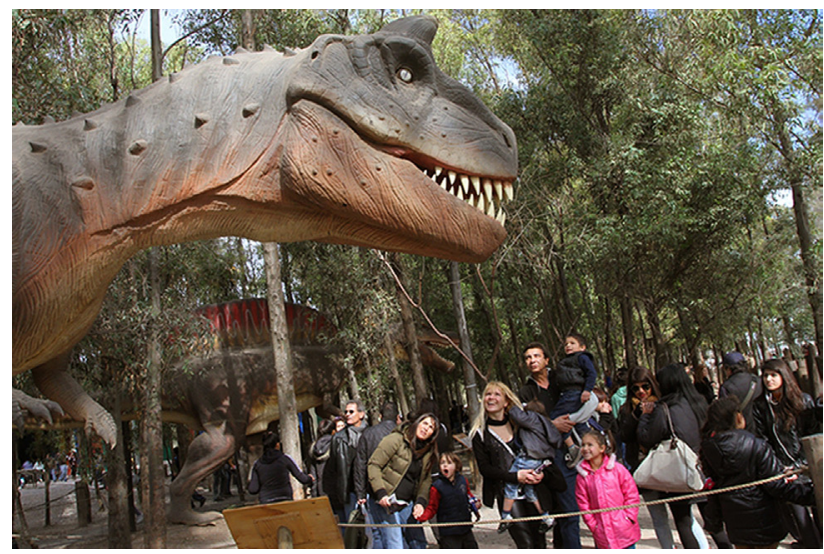

Figura 2: Parque Jurásico en Tecnópolis

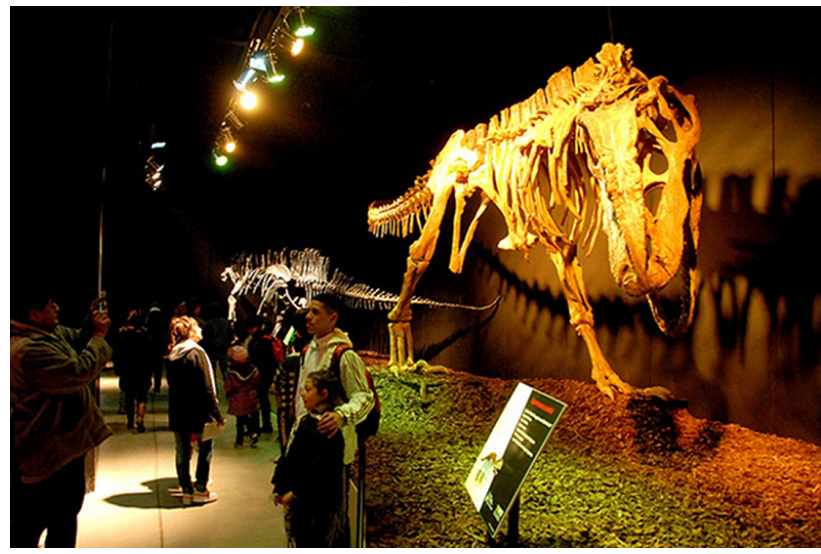

Figura 3: Muestra Paleontología en Argentina.

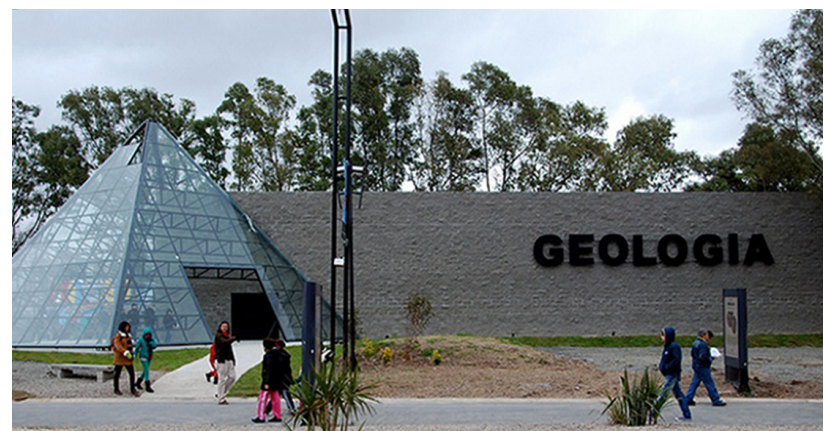

Figura 4: Stand de YPF

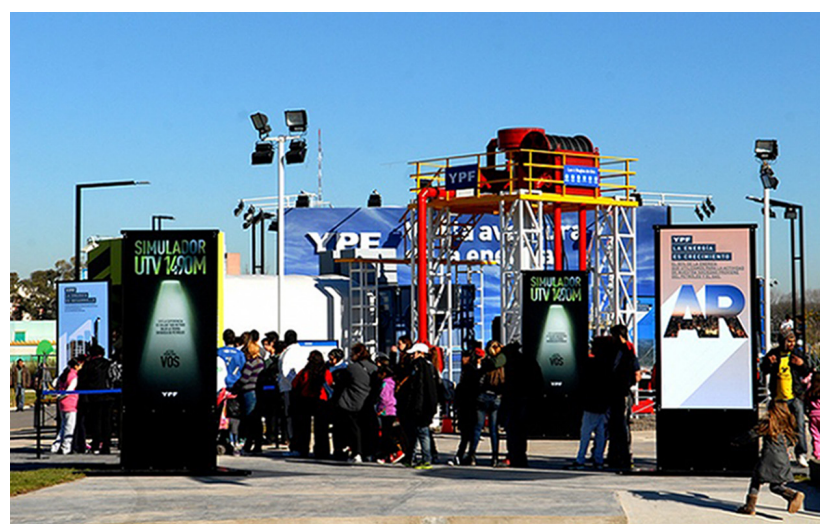

Figura 5: Stand de Geología 
microprogramas que se relacionan con las Ciencias de la Tierra:

\section{Científicos en la Antártida}

Esta serie de microprogramas, de unos pocos minutos de duración cada uno, presenta el trabajo que desarrollan los científicos argentinos en el suelo antártico. A través de un recorrido por su imponente paisaje, el espectador es introducido en ese territorio y conoce los científicos que allí trabajan. http://www.tectv.gob.ar/index.php/micros/component/content/article/77-micros/161-cientificos-en-la-antartida

\section{Futuros}

Se trata de microprogramas en los que se plantea la decisión vocacional de qué carrera estudiar en la universidad. En 3 minutos se presentan varios de los testimonios de egresados y estudiantes de carreras que son de interés estratégico para el país. Se aborda específicamente qué se estudia, dónde, con qué objetivos, cuáles son sus proyectos de investigación y de trabajo. Se relacionan los estudios a seguir con el crecimiento, el desarrollo y el futuro de Argentina. En relación con las Ciencias de la Tierra se presentan las carreras de Geología, Ciencias de la Atmósfera y Paleontología.

http://www.tectv.gob.ar/index.php/micros/component/content/article/77-micros/280-futuros

Ciencias de la Atmósfera http://www.youtube.com/ watch?v=KIOiW3YXAIM

Geología http://www.youtube.com/watch?v $=$ AyU3oybJerU

Paleontología http://www.youtube.com/ watch?v= Gb6NqKpRn8c

El canal se propone motivar a los jóvenes a proseguir carreras científicas. En general, presenta sus productos con una visión neutral de la actividad científica.

\section{3.- Canal de Televisión Encuentro}

Esta señal es la primera señal abierta de televisión del Ministerio de Educación de la Nación. Comenzó su transmisión el 5 de marzo de 2007. Si bien se dirige a todo el público, constituye una importante herramienta para la comunidad educativa. Es un canal federal que incluye contenidos de todas las regiones de la Argentina, además de producciones adquiridas de productoras de América Latina y del mundo.
Se trata de un servicio público de comunicación y no posee publicidad. Este canal llega a seis millones de hogares de todo el país, durante las veinticuatro horas del día. Puede verse en vivo a través de internet: www.encuentro.gov.ar, posibilitando el acceso a los contenidos por parte de otros países de Latinoamérica y el mundo.

Encuentro en internet complementa la emisión televisiva y vincula la transmisión con las nuevas tecnologías de la información y la comunicación para potenciar ambos medios y generar un espacio de convergencia. El sitio de internet posee información sobre programación, recursos educativos multimedia e interactivos, noticias y novedades institucionales, propuestas audiovisuales especialmente pensadas para su uso on line y acceso a otros sitios de interés educativo. Algunos recursos relacionados con las Ciencias de la Tierra son:

\section{La historia del petróleo en Argentina}

Esta serie documental cuenta con 8 capítulos que relatan los inicios de la industria petrolera en Argentina, su evolución y los impactos sufridos por las distintas políticas aplicadas desde el Estado en cada etapa de la historia nacional, desde su nacimiento hasta la política ejercida en la década de 1990 en la que se vendió la empresa petrolera de bandera.

http://www.encuentro.gov.ar/sitios/encuentro/programas/ver?rec_id $=101883$

\section{La historia de la Tierra}

Un documental que propone descubrir los secretos del planeta Tierra y realizar un viaje de descubrimiento científico por todo el planeta. La actividad del núcleo terrestre, la vida en los fondos oceánicos, entre otras grandes temáticas . - http:// www.encuentro.gov.ar/sitios/encuentro/programas/ ver?rec_id=113615\#sthash.rc1aSZwG.dpuf

\section{Naturalistas viajeros}

Este material exhibe los viajes de exploración de los grandes naturalistas que exploraron la Argentina durante los siglos XVIII y XIX. Figuras de la talla de Darwin, Hudson, Moreno o los hermanos Ameghino forman parte de las historias que se cuentan. Esta serie fue producida por la Fundación de Historia Natural Félix de Azara. http:// www.encuentro.gov.ar/sitios/encuentro/programas/ ver?rec_id $=101720$ 


\section{Cambio Climático}

Este documental desarrolla la problemática relativa al cambio climático, los efectos del calentamiento global, los cambios en los vientos y las precipitaciones, el deshielo, la deforestación. También se discuten los efectos de este fenómeno sobre la salud, el turismo, el aprovechamiento de la energía, el caso de la Antártida y la producción agropecuaria. Se propone no solo conocer las razones de estos temas y sus efectos sobre la naturaleza y la vida cotidiana, sino también promover una modificación de conductas y hábitos que ayudarán a mitigar sus efectos negativos. http://www.encuentro.gov. ar/sitios/encuentro/programas/ver?rec_id $=50434$

\section{El planeta azul}

Esta propuesta aborda la historia de la naturaleza de los océanos. Abarca aspectos diversos, desde las playas y lugares de escasa profundidad hasta los misterios de las inexploradas fosas marinas. Es una serie de la BBC que mediante los progresos en la fotografía submarina abrieron las puertas a territorios desconocidos nunca antes explorados.

Otros sitios de interés en relación con las Ciencias de la Tierra que propone el Canal Encuentro son:

http://www.encuentro.gov.ar/sitios/encuentro/programas/ver?rec_id $=104036$

http://www.encuentro.gov.ar/sitios/encuentro/programas/ver?rec_id $=102365$

\section{4.- Canal de Televisión Pakapaka}

Es el primer canal infantil público y educativo del Ministerio de Educación de la Nación. Esta nueva señal infantil desarrolla contenidos de alta calidad orientados a educar y a entretener, está abierto a la cultura de todos los sectores. Se apunta a crear una programación de alta calidad que respete los derechos humanos de los niños y niñas, que estimule su creatividad e imaginación, que promueva la diversidad y la inclusión y que despierte el gusto por el conocimiento. También se propone poner a disposición de docentes y alumnos material audiovisual educativo de alta calidad técnica y pedagógica destinado a apoyar el proceso de enseñanza y aprendizaje en diversas áreas curriculares y favorecer el uso de las tecnologías de información y comunicación en los procesos pedagógicos. En especial, hay un programa desarrollado en relación las Ciencias de la Tierra:

\section{Vuelta por el universo}

Se trata de un dibujo animado cuyos protagonistas --Gabriela, Manuel y Leonardo-- ponen en funcionamiento un cohete que los lleva al espacio. En sus intentos por regresar a la Tierra llegan a diferentes planetas y mundos extraños. Acompañados por la computadora de la nave los chicos conocerán una diversidad de características y datos de la Tierra y de otros lugares del Universo.

Por ejemplo "Continentes" es un capítulo en el que se da una explicación simple y concreta sobre la teoría de tectónica de placas: http:// www.pakapaka.gob.ar/sitios/pakapaka/videos/ index? reproducir $=119133$

\section{5.- Programa Conectar Igualdad}

El Programa Conectar Igualdad, creado en el año 2010, es una política de Estado que busca recuperar y valorizar la escuela pública con el fin de reducir las brechas digitales, educativas y sociales en todo el país. A través de este programa se distribuyen netbooks para los estudiantes y docentes de las escuelas secundarias públicas, de escuelas de educación especial y de institutos de formación docente. Hasta diciembre del 2013 se han distribuido más de 3.800 .000 netbooks. El programa contempla el uso de las netbooks en el ámbito escolar y en el hogar de modo tal de lograr un impacto en la vida diaria de las familias menos pudientes y de las más heterogéneas comunidades de la Argentina.

http://www.conectarigualdad.gob.ar/

En el denominado Escritorio del Docente se ofrecen recursos para enseñar contenidos que se vinculan con propuestas didácticas para implementar en las aulas. Algunos recursos relacionados con las Ciencias de la Tierra, son por ejemplo el trabajo con Google Earth y Google Maps, el proceso de fosilización, la minería, los glaciares, los océanos, el agua subterránea, entre otras posibilidades a las que se puede acceder a través de su página de internet: http://www.conectarigualdad.gob.ar/seccion/ docentes/escritorio-del-docente-42

\section{6.- Semana Nacional de la Ciencia y la Tecnología}

Es una iniciativa impulsada por el Ministerio de Ciencia, Tecnología e Innovación Productiva, a través de su Programa Nacional para la Popularización de la Ciencia y la Innovación. 
Anualmente, museos, centros de investigación, bibliotecas, academias de ciencia, jardines botánicos, universidades, clubes de ciencia, cines y teatros ofrecen un conjunto de actividades para que estudiantes, docentes y público en general se acerquen a la ciencia. Las actividades son gratuitas, se desarrollan en todo el país y abarcan talleres temáticos, charlas con especialistas, visitas guiadas a laboratorios de investigación, muestras de experimentos, exposiciones fotográficas, cine científico y visitas de investigadores del sistema nacional de ciencia y tecnología a las escuelas.

Su página de internet es: http://www.semanadelaciencia.mincyt.gob.ar/

\section{7.- Semana de las Ciencias de la Tierra}

Se trata de una actividad de extensión universitaria realizada por la Facultad de Ciencias Exactas y Naturales de la Universidad de Buenos Aires. Suele tener una duración de entre 3 y 4 días. Se realiza desde el año 2001 y sus actividades más destacadas son: charlas de popularización científica, realización de experimentos demostrativos, exposiciones, visitas guiadas a laboratorios, clases $\mathrm{y}$ talleres interactivos, mostraciones, explicaciones de posters, maquetas, simulaciones y numerosas experiencias didácticas. El horizonte que se pretende alcanzar es principalmente el de compartir conocimiento entre quienes lo enseñan y lo crean y despertar vocaciones.

Este emprendimiento forma parte de un programa muy amplio de extensión universitaria destinado a la popularización del conocimiento científico. Son muchas las instancias que abarca, entre ellas, la Semana de las Ciencias de la Tierra. La información está disponible en:

https://www.facebook.com/PopularizacionExactasUBA/

Todas estas propuestas pueden considerarse recursos de popularización o de enseñanza de las Ciencias de la Tierra pero es sumamente importante definir qué objetivos se persiguen en su instrumentación. Es importante no ofrecer una visión descontextualizada de la ciencia y de sus aplicaciones tecnológicas, incluyendo sus aspectos críticos.

\section{6.- Reflexiones}

La política científica argentina actual otorga un lugar destacado a la popularización y la educación en ciencias, en especial en Ciencias de la Tierra. Las acciones descriptas en este trabajo no representan ni a todas las instituciones ni todas sus acciones pero dan testimonio del momento histórico que atraviesa la Argentina en relación con la promoción de la actividad científica, en general, y de las Ciencias de la Tierra, en particular.

Otro aspecto importante a destacar, refiere a la confluencia latinoamericana pues los países de la región buscar generar políticas coincidentes en diferentes aspectos para potenciar regionalmente tanto la generación de conocimiento científico como su aplicación a la generación de valor agregado en las materias primas que normalmente se exportan desde la región hacia los llamados países desarrollados. Este aspecto es central en la política argentina de popularización pues es un objetivo clave mostrar qué ciencia se hace en el país y cómo su desarrollo promueve el crecimiento de la nación y, con ello, de sus ciudadanos.

Parte de la información de las Ciencias de la Tierra que expusimos en este trabajo refiere a procesos extractivos, tanto de minerales como de hidrocarburos. Su socialización suele abarcar los aspectos técnicos sin acudir a los aspectos sociales que están involucrados en estos procesos. En especial, se hace poca referencia a los pasivos ambientales que estos procesos conllevan, o si lo hacen suelen ser acríticos (Taddei y Seoane 2010). Los conflictos sociales que se desatan en los pueblos afectados por estas actividades pues no suelen ser consultados sobre su instalación. Muchos de estos conflictos son reprimidos, no generan puestos de trabajos, no son controlados en su extracción ni dejan grandes ganancias. Estos aspectos deberían formar parte de la discusión ciudadana. La didáctica de las ciencias promueve incorporar esta discusión en las aulas.

Los referentes que teorizan sobre la popularización y la educación en ciencias dan cuenta de la necesidad de vincular al ciudadano con las decisiones que subsumen dentro de sí cuestiones científicas, sugiriendo generar instancias en las que se proponga a los sujetos adoptar una actitud crítica frente a los aspectos científicos que se vinculan con su realidad. Esta cuestión tiene dos niveles de anclaje frente a la necesidad de adoptar tal actitud crítica, diferentes: el personal y el social. Estos aspectos a revisar a la hora de definir los objetivos de las acciones que aquí 
presentamos. Sin embargo, es importante dar visibilidad a todos los esfuerzos que se están realizando actualmente para difundir las Ciencias de la Tierra en la sociedad argentina actual..

\section{7.- Agradecimientos}

Este trabajo ha sido posible gracias al aporte de los Proyectos UBACyT 20020110200086BA y UBACyT 20020100100699.

\section{8.- Referencias bibliográficas}

Argentina 2007. Ministerio de Ciencia, Tecnología e Innovación Productiva URL: http://www. mincyt.gob.ar/ministerio-presentacion. Acceso: 18.01.2014.

Castro Moreira, I. 2006. A inclusao social e a popularizacao da ciencia e tecnología. Inclusao Social 1(2). URL: http://revista.ibict.br/inclusao/index.php/ inclusao/article/view/29/50. Acceso: 18.01.2014.

Conectar Igualdad, Programa. URL: http://www.conectarigualdad.gob.ar/. Acceso: 18.01.2014.

Encuentro, Canal de Televisión. URL: : www.encuentro.gov.ar. Acceso: 18.01.2014.

Gil Pérez D., Vilches A. 2006. Educación ciudadana y alfabetización científica: Mitos y Realidades. Revista Iberoamericana de Educación. 42:31-54.

Massarini A., Schnek A., Piccinali R., Folguera G. 2007. Democratizar el conocimiento científico: criterios y estrategias para un cambio en la enseñanza de las ciencias. In: Congreso de comunicación social de la ciencia. Cultura Científica y Cultura Democrática, 6. Actas.... Madrid: 21-23 nov. 2007. URL: http://www.csciencia2007.csic. es/actas/co a3 01.pdf. Acceso: 18.01.2014.

Merino G 2000. De qué hablamos cuando hablamos de la alfabetización científica en al ciudadanía del siglo XXI. En Bottinelli N., Giamello R. eds. 2000. Ciencia, Tecnología y Vida Cotidiana Reflexiones y Propuestas del Nodo Sur de la Red Pop. Uruguay: Nodo Sur de la Red Pop. P. 51-58.

Pakapaka, Canal de Televisión. URL: http://www. pakapaka.gob.ar. Acceso: 18-01-2014.

Ramos V. 2011. Doscientos años de Ciencias de la Tierra en Argentina. Buenos Aires: Rev. Asoc. Geol. Argent. 68(3): URL:http://www.scielo.org. ar/scielo.php? script $=$ sci_arttext $\&$ pid $=$ S0004$-48222011000300011 \& \operatorname{lng}=$ es\&nrm $=$ iso. Acceso: 17-01.2014.

Sahlberg, P. 2013. El cambio educativo en Finlandia ¿qué puede aprender el mundo? Buenos Aires: Paidós. $236 \mathrm{p}$.

Sanmartí N. 2002. Didáctica de las ciencias en la educación secundaria obligatoria. Madrid: Síntesis. 382 p.

Semana de la Ciencias de la Tierra. URL: https://www. facebook.com/PopularizacionExactasUBA Acceso 18.01.2014.

Semana Nacional de la Ciencia y la Tecnología. URL http:// www.semanadelaciencia.mincyt.gob.ar/

Seoane J., Taddei E. 2010. Recolonización, bienes comunes de la naturaleza y alternativas de los pueblos. Río de Janeiro: IBASE. 94p.

Tecnópolis TV. URL http://www.tectv.gob.ar/. Acceso: 18.01.2014.

Tecnópolis: Megamuestra de ciencia, tecnología, industria y arte. URL http://tecnopolis.ar/2013/. Acceso: 18.01.2014. 